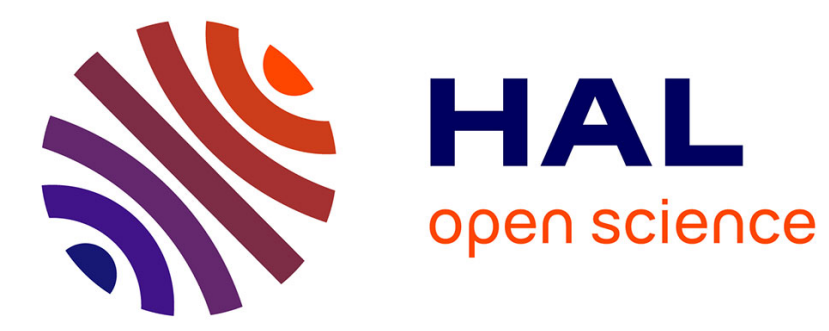

\title{
Stabilization of pulse waves through inhibition in a feedforward neural network
}

Arnaud Tonnelier

\section{To cite this version:}

Arnaud Tonnelier. Stabilization of pulse waves through inhibition in a feedforward neural network. Physica D: Nonlinear Phenomena, 2005, 210, pp.118-137. hal-00390718

\section{HAL Id: hal-00390718 https://hal.science/hal-00390718}

Submitted on 3 Jun 2009

HAL is a multi-disciplinary open access archive for the deposit and dissemination of scientific research documents, whether they are published or not. The documents may come from teaching and research institutions in France or abroad, or from public or private research centers.
L'archive ouverte pluridisciplinaire HAL, est destinée au dépôt et à la diffusion de documents scientifiques de niveau recherche, publiés ou non, émanant des établissements d'enseignement et de recherche français ou étrangers, des laboratoires publics ou privés. 


\title{
Stabilization of pulse waves through inhibition in a feedforward neural network
}

\author{
A. Tonnelier ${ }^{1}$ \\ Cortex Project, INRIA Lorraine, Campus Scientifique, 54 602 Villers-lès-Nancy, France
}

\begin{abstract}
We consider a firing rate model of a neural network of excitatory and inhibitory populations with an excitatory feedforward connectivity. We analyze traveling wave solutions and determine the conditions for their existence and stability. Our study demonstrates the role of inhibition in stable pulse propagation. In a purely excitatory network, pulse waves are unstable because of the existence of stable front wave and back wave with different velocities. Pulse waves can propagate stably in the network where excitation is appropriately balanced by inhibition. Analytical results on the wave speeds and the shape of waves are obtained.
\end{abstract}

\section{Introduction}

Propagation of patterns of neural activity occurs in various neural systems and seems to play an important role for information processing in the brain. Traveling waves of synchronized neural excitation have been observed in various brain regions, including the cortex [12], thalamus [13] and hippocampus [17]. These waves are the consequence of nonlinear synaptic interactions between neurons. Neural tissues are commonly seen as neural fields which form and propagate patterns of excitation [24], [2]. Propagation of waves in neural network has been the subject of several recent theoretical studies [18], [3]. Mathematical analysis of firing rate models can often lead to some general wave properties, such as their propagation velocity and its dependence on model parameters [9], [19], [7].

Two complementary frameworks are commonly used when studying traveling waves of neural activity: spatially structured neuronal networks with continuous connectivity and layered feedforward networks consisting of pools of populations (or neurons). Traveling waves in spatially continuous networks are largely studied [8], [3]. There are now a number of results about the existence and the stability of traveling pulses [25], [5], [10] . Layered feedforward networks can be seen as a discrete version of the former where the smooth propagating wave is replaced by a discrete transmission of activities from one pool to the next [1], [6]. If a layer fires in synchrony then the next layer will also do so and a so called synfire activity will propagate. The capability of feedforward networks composed of discrete pools to transmit rate code have been analyzed [15], [14]. The existence of negative feedbacks such as inhibition or refractory behavior are crucial for the propagation of pulses. Networks without negative feedbacks only exhibit front wave whereas traveling pulse fails to propagate. There are a growing number of papers concerned with propagating pulses in networks with inhibition or with some form of adaptation [19], [10]. The present work aims at a better understanding of propagation in feedforward neural networks consisting of discrete pools and clarifies the role of inhibition in propagating waves. In particular, we raise the following theoretical questions:

\footnotetext{
${ }^{1}$ Tel.:+33 383593072 ; fax:+33 383413079

E-mail address: arnaud.tonnelier@loria.fr
} 
(1) How is the propagation supported by excitatory synapses in layered feedforward networks? What are the effects of inhibition?

(2) How does the propagation depend on the various parameters including the synaptic strengths, the time constants?

To answer these questions, we consider a simple approach using a firing rate description. Time-dependent firing rate models are commonly described by

$$
\tau \frac{d r}{d t}=-r+F(W \cdot r+h)
$$

where $r$ is the firing rate vector, $\tau$ the relaxation time, $W$ is the matrix of dimensionless synaptic weights and $h$ an external input. The function $F$ is the so-called activation function that describes the steady-state firing rate as a function of the input current. A common choice is the sigmoidal function

$$
F\left(I_{s}\right)=\frac{1}{1+e^{-\lambda\left(I_{s}-\theta\right)}}
$$

where $\theta$ is a threshold value and $\lambda>0$ determines how rapidly the firing rate increases as a function of $I_{s}$. In the high gain limit $\lambda \rightarrow \infty$ the activation function is rewritten as

$$
F\left(I_{s}\right)=\Theta\left(I_{s}-\theta\right)
$$

where $\Theta$ the Heaviside step function defined by $\Theta(s)=0$ for $s<0$ and $\Theta(s)=1$ for $s>0$. We consider a network of excitatory (E) and inhibitory (I) populations. Each population is described by its firing rate $r_{e}$, $r_{i}$, for the excitatory and inhibitory population, respectively. The dynamics is governed by a coupled set of equations identical in form to (1),

$$
\begin{aligned}
\tau_{e} \frac{d r_{e}}{d t} & =-r_{e}+\Theta\left(w_{e e} r_{e}+w_{i e} r_{i}+h_{e}-\theta_{e}\right), \\
\tau_{i} \frac{d r_{i}}{d t} & =-r_{i}+\Theta\left(w_{i i} r_{i}+w_{e i} r_{e}-\theta_{i}\right),
\end{aligned}
$$

where we allow the excitatory and inhibitory populations to have different time constants $\tau_{e}, \tau_{i}$ and different thresholds $\theta_{e}, \theta_{i}$, respectively. The synaptic weights $w_{\alpha \beta}, \alpha=e, i$ and $\beta=e, i$, describe the four possible types of neuronal interactions within a pool and $h_{e}$ is an external input. Synaptic weights from the excitatory population are positive whereas those from the inhibitory population are negative. We consider a one-dimensional network with discrete nearest-neighbor coupling with the external input received by the $k^{\text {th }}$ excitatory population given by

$$
h_{e, k}(t)=w_{f} r_{e, k-1}(t)
$$

where $w_{f}$ is the weight connection and $r_{e, k}$ is the firing rate activity of the excitatory population located at the $k^{\text {th }}$ pool (see Fig. 1). Despite its very restrictive architecture, the network shows nontrivial dynamics. The major advantage of the model is the exact solvability of the equations that allows us to derive analytical conditions for the existence and stability of propagating waves. Multilayer architectures with purely feedforward connectivity have been used to explore information transfer in biological networks [23]. Such architecture without recurrent connections is relevant when studying fast sensory processing where input from feedback connections can be neglected in the early response.

We explore the propagation properties of the neural network using both mathematical analysis and numerical simulations. Our analysis provides an analytical characterization of the influence of all the parameters of the model including the synaptic weights, the inhibitory and excitatory relaxation times and the thresholds. Taking the advantage of the idealized response function we calculate exactly the velocity and stability of propagating 
traveling waves. We investigate analytically the role played by inhibition in the existence and stability of propagating pulses.

The paper is organized as follows. The dynamics of an isolated pool, i.e. the uncoupled network $w_{f}=0$, is analyzed in section 2. We use the bifurcations that occur in an isolated pool to understand the propagating waves described in section 3. The main emphasis is given to traveling pulses. To clearly illustrate the role of inhibition we distinguish between excitatory network and network with a balance of excitation and inhibition. We conclude by a discussion.

\section{The two population model}

We analyze the dynamics of a single pool. We take the advantage of the piecewise linear function (3) to derive the analytical expressions of the bifurcations that occur in the model. An isolated pool is described by the two scalars $r_{e}(t)$ and $r_{i}(t)$ that follow the set of coupled equations (4). For the sake of simplicity, we neglect the self-inhibition of the inhibitory population by taking $w_{i i}=0$. This does not change the basic properties of the model but simplifies the results and their interpretation. Since we consider the excitatory coupled network, i.e. synaptic interactions between the excitatory populations and no long range connection for the inhibitory one, we consider $h_{e}$ as a bifurcation parameter. It is convenient to distinguish two cases depending on the relative strength of the excitatory-inhibitory synaptic connection $w_{e i}$ and the inhibitory firing threshold $\theta_{i}$. When $w_{e i}<\theta_{i}$ the inhibition does not play a role since the excitatory input $w_{e i} r_{e}$ cannot reach the threshold $\theta_{i}$ and thus the inhibitor field cannot be activated. In the following, we say excitatory network when $w_{e i}<\theta_{i}$ and balanced network otherwise.

Excitatory network. In this case, the study of a pool reduced to the excitatory population, $\tau_{e} d r_{e} / d t=$ $-r_{e}+\Theta\left(w_{e e} r_{e}+h_{e}-\theta_{e}\right)$. It is straightforward to show that $r_{e}=0$ is a stable fixed point as long as $h_{e}<\theta_{e}$ and a saddle node bifurcation, noted SN1, occurs at

$$
h_{e e}^{S N 1}=\theta_{e}-w_{e e}
$$

where the stable high activity state $r_{e}=1$ appears. Due to the Heaviside step function the dynamical system has a discontinuous right-hand side and a precise definition of the saddle node bifurcation requires additional comments that are beyond the scope of this paper [11]. Since the saddle point, that lies on the line of discontinuity, does not play a role in our analysis, we will not characterize it. For $h_{e e}^{\mathrm{SN} 1}<h_{e}<\theta_{e}$, two stable states coexist until $h_{e}$ reaches the saddle node bifurcation (SN2)

$$
h_{e}^{S N 2}=\theta_{e}
$$

where the low-activity state $r_{e}=0$ disappears leading to the purely excitatory steady state.

Balanced network. The two threshold lines $w_{e e} r_{e}+w_{i e} r_{i}=\theta_{e}-h_{e}$ and $w_{e i} r_{e}=\theta_{i}$ delimit the domains where the system is linear. Using a geometrical analysis in the phase plane $\left(r_{e}, r_{i}\right)$ (see Fig. 2B) we easily derive that the steady state $\left(r_{e}, r_{i}\right)=(0,0)$ exists while $h_{e}<\theta_{e}$ and an additional steady state $\left(r_{e}, r_{i}\right)=(1,1)$ appears when $h_{e}>h_{e i}^{S N 1}$ where

$$
h_{e i}^{S N 1}=\theta_{e}-w_{i e}-w_{e e} .
$$

Its location depends on the relative strength between excitation and inhibition. Note that $h_{e}^{S N 2}$ given by (5) is still a bifurcation point leading to the disappearance of the low activity steady state.

Summarizing, the isolated pool has two saddle-node bifurcations that monitor the dynamics. The bifurcation diagram of the system is shown in Fig. 2A. The two bifurcations are closely connected due to the symmetry property of eq. (4). In fact, the system is invariant under the change of variables $r_{e} \rightarrow 1-r_{e}, r_{i} \rightarrow 1-r_{i}$ combined with $h_{e} \rightarrow 2 \theta_{e}-w_{e e}-h_{e}$ for the excitatory network and $h_{e} \rightarrow 2 \theta_{e}-w_{e e}-w_{i e}-h_{e}, \theta_{i} \rightarrow w_{e i}-\theta_{i}$ for 
the balanced network (with $w_{i i}=0$ ). As we will show in the next section, the bifurcations are closely related to the traveling waves that occur in the connected network. Moreover the symmetry of the two bifurcation points leads to remarkable properties of the waves.

\section{$3 \quad$ Firing rate propagation in a chain of pools}

We consider a chain of identical pools with feedforward connectivity. The firing rate activity of the pool locate at $k \in Z$ is described by

$$
\begin{aligned}
\tau_{e} \frac{d r_{e, k}}{d t} & =-r_{e, k}+\Theta\left(w_{e e} r_{e, k}+w_{i e} r_{i, k}+w_{f} r_{e, k-1}-\theta_{e}\right), \\
\tau_{i} \frac{d r_{i, k}}{d t} & =-r_{i, k}+\Theta\left(w_{e i} r_{e, k}-\theta_{i}\right) .
\end{aligned}
$$

The network (6) has two trivial traveling wave solutions that are standing waves given by the two following homogeneous stationary solutions: the homogeneous non firing state that is the "all-off" state $\left(r_{e, k}, r_{i, k}\right)_{k}=$ $(0,0)_{k}$ and the "all-on" state $\left(r_{e, k}, r_{i, k}\right)_{k}=(1,1)_{k}$. Both are stable regarding small perturbations: for $r_{e, k}(t)=$ $r_{e, s t a t}+\delta_{e, k}, r_{i, k}(t)=r_{i, \text { stat }}+\delta_{i, k}$ where $r_{\text {stat }}$ is an homogeneous solution and $\left|\delta_{e, k}\right| \ll 1,\left|\delta_{i, k}\right| \ll 1$, we obtain the time evolution $d \delta_{e, k} / d t=-\delta_{e, k}, d \delta_{i, k} / d t=-\delta_{i, k}$. The "all-on" solution exists for excitatory network if $w_{e e}+w_{f}>\theta_{e}$ and $w_{e e}+w_{f}+w_{i e}>\theta_{e}$ for balanced network.

Formally, a traveling wave is a solution of (6) such that

$$
\begin{aligned}
& r_{e, k}(t)=R_{e}(t-k / c), \\
& r_{i, k}(t)=R_{i}(t-k / c)
\end{aligned}
$$

where $c$ is the wave velocity and $R_{e}, R_{i}$, the wave function of excitatory, inhibitory, population, respectively. Inserting (7) into (6), the wave functions satisfy

$$
\begin{aligned}
\tau_{e} \frac{d R_{e}}{d \xi} & =-R_{e}+\Theta\left(w_{e e} R_{e}+w_{i e} R_{i}+w_{f} R_{e}(\xi+1 / c)-\theta_{e}\right) \\
\tau_{i} \frac{d R_{i}}{d \xi} & =-R_{i}+\Theta\left(w_{e i} R_{e}-\theta_{i}\right)
\end{aligned}
$$

where $\xi=t-k / c$ is the traveling wave coordinate. We will mainly focus on pulse wave, that is a traveling wave solution that connects the homogeneous steady state $(0,0)_{k}$ to itself. The properties of pulse wave are closely related to the existence of two other types of waves: front wave and back wave that connect two different homogeneous stationary solutions. These two waves differ from the boundary conditions at $\pm \infty$; wave front advances the "on" state from left to right whereas wave back also referred as retracting wave advances the "off" state.

Numerically, traveling waves are initiated using an injected current into a group of pools at the beginning of a finite chain. Starting from the low activity steady state, it is clear from (6) that a necessary condition for the propagation of an excitatory activity reads

$$
w_{f}>\theta_{e}
$$

i.e. the activity of a pool is able to initiate an activity in its neighboring pool. In other words, a propagation of an excitatory activity is possible if the coupling strength is sufficiently large and at least greater than the excitatory 
threshold $\theta_{e}$. The time-dependent input $w_{f} r_{e, k-1}$ can be related to the bifurcation parameter $h_{e}$ previously used (section 2). The saddle-node bifurcation of the single pool model leading to the disappearance of the low activity steady state is then associated to the propagation condition since (9) could be rewritten $w_{f}>h_{e}^{\mathrm{SN} 2}$. The velocity of the propagating front is given by the time to reach the saddle node bifurcation SN2. Indeed, let $t_{k-1}^{\text {th }}$ be the time at which the activity induced by the pool $k-1$ reaches the threshold, i.e. $w_{f} r_{e, k-1}=\theta_{e}$, then the time course of the firing rate activity of the postsynaptic pool is given by $r_{e, k}(t)=1-\exp \left(-\left(t-t_{k-1}^{\mathrm{th}}\right) / \tau_{e}\right)$. The propagation speed is defined by $c_{f}=1 /\left(t_{k}^{\text {th }}-t_{k-1}^{\text {th }}\right)$ and we calculate

$$
c_{f}=\left[\tau_{e} \ln \left(w_{f} /\left(w_{f}-\theta_{e}\right)\right)\right]^{-1} .
$$

The time interval $1 / c_{f}$ is the time required for $w_{f} r_{e}$ to go from 0 to $\theta_{e}$ and thus represents the time to reach the bifurcation point $h_{e}^{\mathrm{SN} 2}$. Note that the speed of a propagating activity is only determined by the initial wave front and does not depend on its profile. Therefore traveling fronts and traveling pulses have the same velocity. Of particular interest is the speed of the propagating front for small and high values of the coupling strength. In the limit of a strong connection $w_{f} \rightarrow+\infty$ the velocity follows the linear law $c_{f} \rightarrow w_{f} /\left(\tau_{e} \theta_{e}\right)$ and at the inset of propagation obtained as $w_{f} \rightarrow \theta_{e}^{+}$the velocity follows the logarithmic law $c_{f} \rightarrow 1 /\left(\tau_{e}\left|\ln \left(w_{f}-\theta_{e}\right)\right|\right)$. The precise conditions for the existence of a traveling wave solution and the determination of its profile is monitored by the interplay between the excitatory and inhibitory population. We first concentrate on the excitatory network that allows us to introduce the mathematical tools and techniques. The analysis of the balanced network is mathematically similar and is done in a separate section. Basically, we take the advantage of the threshold function (3) observing that $F\left(I_{s}\right)$ depends on the time at which $I_{s}$ reaches the threshold and not on the shape of $I_{s}$.

\subsection{Excitatory network}

In this section, we consider the case of an excitatory network. The inhibitory population does not affect the excitatory one that is formally equivalent to take $w_{i e}=0$. Traveling wave solution are given by eq. (8) with $R_{i}(\xi)=0$. Solutions can be easily calculated if we know the intervals where the total input exceeds the threshold. Let $\xi=0$ be the point where the total input received by an excitatory pool reaches $\theta_{e}$. The front of the wave is given by

$$
R_{e}(\xi)=\left(1-e^{-\xi / \tau_{e}}\right) \Theta(\xi) .
$$

When $w_{f}+w_{e e}>\theta_{e}$ holds the excitatory all-on state exists and therefore the excitation induced by the propagating front persists. Since $w_{f}>\theta_{e}$ is a necessary condition for the existence of a propagating activity then the traveling front solution given by (11) exists as long as a propagation of an excitatory activity is possible. Traveling pulse appears when the front is followed by a return to the low-activity resting state, i.e. we require the boundary conditions $\lim _{ \pm \infty} R_{e}=0$. We thus request that the interval where $R_{e}$ increases is bounded: let $\left(0, \xi_{e}^{1}\right)$ be the interval where the totel input $w_{e e} R_{e}(\xi)+w_{f} R_{e}(\xi+1 / c)$ exceeds the threshold $\theta_{e}$ (see Fig. 3). A traveling pulse solution is given by

$$
\begin{aligned}
& \tau_{e} R_{e}^{\prime}=-R_{e}, \quad \text { for } \xi \in(-\infty, 0) \cup\left(\xi_{e}^{1},+\infty\right), \\
& \tau_{e} R_{e}^{\prime}=-R_{e}+1, \quad \text { for } \xi \in\left(0, \xi_{e}^{1}\right) .
\end{aligned}
$$

The excitatory wave function $R_{e}$ increases for $0 \leq \xi \leq \xi_{e}^{1}$ and decreases for $\xi_{e}^{1} \leq \xi \leq \infty$. The threshold condition at $\xi=0$ reads $w_{f} R_{e}(1 / c)=\theta_{e}$ that gives the wave speed previously obtained in (10). The parameter $\xi_{e}^{1}$ satisfies

$$
w_{e e} R_{e}\left(\xi_{e}^{1}\right)+w_{f} R_{e}\left(\xi_{e}^{1}+1 / c_{f}\right)=\theta_{e}, \xi_{e}^{1}>0 .
$$


Using the expression of the traveling pulse derived from (12) combines with the expression of the velocity gives

$$
\xi_{e}^{1}=\tau_{e} \ln \left(\left(w_{e e}+w_{f}-\theta_{e}\right) /\left(w_{e e}+w_{f}-2 \theta_{e}\right)\right) .
$$

A traveling pulse exists if the branching point $\xi_{e}^{1}$ exists. From (9) we have $w_{e e}+w_{f}-\theta_{e}>0$ and thus we need $w_{e e}+w_{f}-2 \theta_{e}>0$. We obtain the following condition

$$
w_{e e}+w_{f}>2 \theta_{e}
$$

Moreover our analysis requires the consistency condition $\xi_{e}^{1}>1 / c_{f}$ to ensure that the presynaptic population does not cross down through $\theta_{e}$ before the postsynaptic population reaches up to $\theta_{e}$. That gives

$$
w_{e e}<\theta_{e} .
$$

Under the two conditions (13),(14), the traveling pulse is given by

$$
R_{e}(\xi)=\left(1-e^{-\xi / \tau_{e}}\right) \Theta(\xi)+\left(e^{-\left(\xi-\xi_{e}^{1}\right) / \tau_{e}}-1\right) \Theta\left(\xi-\xi_{e}^{1}\right) .
$$

As we will show, the traveling pulse is unstable and thus cannot propagate stably in the network. To study the stability we go back to the network equations (6). Let $t_{k}$ be the pulse width of the $k^{\text {th }}$ excitatory population defined as the time interval during which the total input received by the $k^{\text {th }}$ population is superthreshold. In other words, $t_{k}$ is the interval between two successive threshold reaching for the total input of the pool located at $k$. This time interval is defined by

$$
w_{e e} r_{e, k}\left(t_{k}\right)+w_{f} r_{e, k-1}\left(t_{k}\right)=\theta_{e}
$$

where the rise time of $r_{e, k}$ is used as the origin of time, i.e. $r_{e, k}(t)=1-e^{-t / \tau_{e}}$. In the decaying phase the activity of the presynaptic pool follows $r_{e, k-1}(t)=C e^{-\left(t+1 / c_{f}\right) / \tau_{e}}$ where $C$ is a constant and the time is shifted forward by $1 / c_{f}$ that is the needed time to initiate the activity of a pool when the presynaptic one is activated. The matching condition at $t_{k-1}$ gives $r_{e, k-1}(t)=\left(e^{t_{k-1} / \tau_{e}}-1\right) e^{-\left(t+1 / c_{f}\right) / \tau_{e}}$. We obtain that the successive intervals are given by

$$
t_{k}=\tau_{e} \ln \left(\frac{\left(w_{f}-\theta_{e}\right)\left(e^{t_{k-1} / \tau_{e}}-1\right)-w_{e e}}{\theta_{e}-w_{e e}}\right)=f\left(t_{k-1}\right) .
$$

A fixed point of the iterated map (15) is defined by $f\left(t^{*}\right)=t^{*}$ and gives the rising interval of the traveling pulse solution $t^{*}=\xi_{e}^{1}$. Its stability is related to the stability of the corresponding traveling pulse. More precisely, it gives the evolution through the network of a perturbation on the shape of the wave. Thus, we restrict ourselves to stability analysis based upon perturbations traveling at the same speed as the wave. This approach is similar to the stability analysis based on perturbations of the firing times introduced in [3]. The traveling pulse solution is stable if $\left|f^{\prime}\left(t^{*}\right)\right|<1$. We calculate

$$
f^{\prime}\left(t^{*}\right)=\frac{\left(w_{e e}+w_{f}-\theta_{e}\right)\left(w_{f}-\theta_{e}\right)}{\theta_{e}\left(w_{f}-\theta_{e}\right)-w_{e e}\left(w_{e e}+w_{f}-2 \theta_{e}\right)} .
$$

that gives

$$
f^{\prime}\left(t^{*}\right)=\frac{w_{f}-\theta_{e}}{\theta_{e}-w_{e e}}
$$

We have $f^{\prime}\left(t^{*}\right)>0$ and it is easy to show that $f^{\prime}\left(t^{*}\right)<1$ if and only if $\left.w_{e e}+w_{f} \in\right] \theta_{e}, 2 \theta_{e}[$. Since the existence of a traveling pulse requires $w_{e e}+w_{f}>2 \theta_{e}$, we have $f^{\prime}\left(t^{*}\right)>1$, i.e. the traveling pulse is unstable. 
The iterated map (15) gives the evolution through the network of an initial pulse and thus allows a characterization of the propagating activity. In particular, the quantity $f(t)-t$ tends asymptotically towards

$$
f(t)-t \sim \tau_{e} \ln \frac{w_{f}-\theta_{e}}{\theta_{e}-w_{e e}}
$$

and thus at each pool the pulse grows by a fixed amount. For an initial pulse width greater than $t^{*}$ the pulse profile increases leading to an enlarging pulse. The speed of the wake tends towards $c_{b}=\left(f(t)-t+1 / c_{f}\right)^{-1}$ as $t \rightarrow \infty$ and we calculate

$$
c_{b}=\left[\tau_{e} \ln \left(w_{f} /\left(\theta_{e}-w_{e e}\right)\right)\right]^{-1} .
$$

Depending on the initial excitation the propagation fails or leads to an enlarging pulse (see Fig. 4). Note that in the limit $w_{e e}+w_{f} \rightarrow 2 \theta_{e}$ we have $f^{\prime}\left(t^{*}\right) \rightarrow 1$ and thus the transition from a traveling pulse (constant shape) to a growing pulse could be slow. Note also that the existence of an enlarging pulse requires $c_{f}>c_{b}$ that gives the condition (13) previously derived .

The propagation of a growing pulse is closely related to the existence of a back wave. When $w_{e e}<\theta_{e}$ the excited state of a postsynaptic pool does not persist if the activity of the presynaptic pool is turned off. Thus there exists a propagation that connects $r_{e}=1$ to $r_{e}=0$. The study of the traveling back could be deduced from the traveling front. In fact, similarly to the single pool model, a symmetry property also holds for the traveling wave solutions. Equation for $R_{e}(\xi)$ is invariant with respect to the transformation $R_{e} \rightarrow 1-R_{e}$ and $\theta_{e} \rightarrow w_{e e}+w_{f}-\theta_{e}$ so the profile of the back wave can be obtain from (11) and the velocity (16) from (10) by replacing $\theta_{e}$ by $w_{e e}+w_{f}-\theta_{e}$. We find that the profile of a back wave is given by

$$
R_{e}(\xi)=1+\left(e^{-\xi / \tau_{e}}-1\right) \Theta(\xi)
$$

and the velocity is the one of the wake of the enlarging pulse $c=c_{b}$. The regime where traveling front and traveling back coexist is the regime where enlarging pulse occurs (see Fig. 5). In this regime we have $c_{f}>c_{b}$ and the enlarging pulse can be obtained as the merging of traveling front and traveling back. Note that the existence of a back wave can be interpret in term of bifurcation. The back front is induced by the saddle-node bifurcation $h_{e e}^{S N 1}$ previously described that leads to the disappearance of the excited state. The time to reach this saddle node also gives the speed of propagation $c_{b}$.

The main results of this section are summarized in Fig. 5

\subsection{Balanced network}

In the previous section, we have assumed that $w_{e i}$ is sufficiently small so that inhibition does not occur. In a balanced network, the inhibitory population is activated by the excitatory one and thus interacts through with it. The basic properties of the traveling fronts and the traveling backs remain unchanged and we describe the main results.

As previously noticed, the velocity of the initial front is not affected by inhibition. The profile of the front remains unchanged and its existence is monitored by the boundary condition $R_{e}(+\infty)=1$. We must have $w_{e e}+w_{i e}+w_{f}>\theta_{e}$ that is related to the existence of the all-on state in the balanced network. Note that the condition for propagation $w_{f}>\theta_{e}$ is no longer sufficient to guarantee the existence of a front wave in the balanced network as it is the case in the excitatory network. Complementary to the excitatory wave function $R_{e}$ there exists an inhibitory front $R_{i}$ since the inhibition is activated at $\xi=\xi_{i}^{0}$ where $\xi_{i}^{0}$ satisfies the threshold condition $w_{e i} R_{e}(\xi)=\theta_{i}$. We calculate

$$
\xi_{i}^{0}=\tau_{e} \ln \left(w_{e i} /\left(w_{e i}-\theta_{i}\right)\right) .
$$

The profile of the traveling front of the inhibitory population is given by

$$
R_{i}(\xi)=\left(1-e^{-\left(\xi-\xi_{i}^{0}\right) / \tau_{i}}\right) \Theta\left(\xi-\xi_{i}^{0}\right)
$$


When $w_{e e}+w_{i e}<\theta_{e}$ an isolated unit cannot maintain a high activity and a back wave could propagate. Exploiting the symmetry properties previously noticed, back wave can be deduced from front wave. The back wave is given by (17) for the excitatory population and

$$
R_{i}(\xi)=1+\left(e^{-\left(\xi-\xi_{i}^{1}\right) / \tau_{i}}-1\right) \Theta\left(\xi-\xi_{i}^{1}\right)
$$

for the inhibitory one where $\xi_{i}^{1}$ is the inactivation point of the inhibitory population defined by $\xi_{i}^{1}=\tau_{e} \ln w_{e i} / \theta_{i}$. The speed of the back wave is given by

$$
c_{b}=\left[\tau_{e} \ln \left(w_{f} /\left(\theta_{e}-w_{e e}-w_{i e}\right)\right)\right]^{-1} .
$$

A representative graph of traveling front and traveling back is depicted in Fig. 6. Similarly to the excitatory network, the front wave is related to the successive reaching of the bifurcation point $h_{e}^{S N 2}$ for each pools whereas back wave is associated to the bifurcation point $h_{e i}^{S N 1}$.

We now turn to the main part of our analysis of propagation in balanced network through the study of pulse waves. We require that $R_{e}, R_{i}$, crosses the threshold $\theta_{e}, \theta_{i}$, exactly twice. We note $\xi_{i}^{0}$ and $\xi_{i}^{1}$ the parameters related to the activation and inactivation of the inhibitory population. The traveling pulse is given by

$$
\begin{aligned}
\tau_{e} R_{e}^{\prime} & =-R_{e}, \quad \text { for } \xi \in(-\infty, 0) \cup\left(\xi_{e}^{1},+\infty\right), \\
\tau_{e} R_{e}^{\prime} & =-R_{e}+1, \quad \text { for } \xi \in\left(0, \xi_{e}^{1}\right), \\
\tau_{i} R_{i}^{\prime} & =-R_{i}, \quad \text { for } \xi \in\left(-\infty, \xi_{i}^{0}\right) \cup\left(\xi_{i}^{1},+\infty\right), \\
\tau_{i} R_{i}^{\prime} & =-R_{i}+1, \quad \text { for } \xi \in\left(\xi_{i}^{0}, \xi_{i}^{1}\right) .
\end{aligned}
$$

It is straightforward to solve the equations and to obtain the analytical expressions of the excitatory and inhibitory wave functions. We show in Fig. 7 the profile of these functions. Existence of a pulse wave is given by the existence of $c, \xi_{e}^{1}, \xi_{i}^{0}$ and $\xi_{i}^{1}$ that satisfy the following threshold conditions

$$
\begin{aligned}
w_{e e} R_{e}(0)+w_{i e} R_{i}(0)+w_{f} R_{e}(1 / c) & =\theta_{e} \\
w_{e i} R_{e}\left(\xi_{i}^{0}\right) & =\theta_{i} \\
w_{e e} R_{e}\left(\xi_{e}^{1}\right)+w_{i e} R_{i}\left(\xi_{e}^{1}\right)+w_{f} R_{e}\left(\xi_{e}^{1}+1 / c\right) & \theta_{e} \\
w_{e i} R_{e}\left(\xi_{i}^{1}\right) & =\theta_{i} .
\end{aligned}
$$

The equation (19) gives the velocity $c=c_{f},(20)$ leads to (18) and (22) gives the inactivation of the inhibitory population at

$$
\xi_{i}^{1}=\tau_{e} \ln \left(\left(w_{e i}\left(e^{\xi_{e}^{1} / \tau_{e}}-1\right)\right) / \theta_{i}\right) .
$$

From (21), we derive the main requirement for the existence of a pulse wave that is related to the existence of the inactivation point for the excitatory population given by

$$
\left(w_{e e}+w_{f}-\theta_{e}\right) e^{-\xi_{e}^{1} / \tau_{e}}+w_{i e}\left(w_{e i} /\left(w_{e i}-\theta_{i}\right)\right)^{\tau_{e} / \tau_{i}} e^{-\xi_{e}^{1} / \tau_{i}}=w_{e e}+w_{i e}+w_{f}-2 \theta_{e}
$$

where $\xi_{e}^{1}$ has to fulfill the two a priori assumptions

$$
\left\{\begin{aligned}
1 / c_{f} & <\xi_{e}^{1} \\
\xi_{i}^{0} & <\xi_{e}^{1}
\end{aligned}\right.
$$

The first requirement is a consistence condition for the existence of the wave speed $c_{f}$. The last assumption states that inhibition is activated when the excitatory population is in its rising phase otherwise the stability result of the previous section remains unchanged and a traveling pulse, when it exists, is unstable. Note that the characteristic point of the inhibitory wave $\xi_{i}^{1}$ exists whenever $\xi_{e}^{1}$ exists. Of particular interest is the regime 
of existence and stability of traveling pulses.

The study of the the stability of pulse waves in balanced network is similar to the analysis of section 3.1. We introduce the time interval $t_{k}$ defined by the iteration

$$
t_{k+1}=f\left(t_{k}\right)
$$

that gives the evolution of a pulse, i.e. an arbitrary bounded excited region, through the network, starting from an initial pulse width $t_{0}$. The function $f$ could be expressed through its inverse given by

$$
f^{-1}(t)=\tau_{e} \ln \frac{\theta_{e}-w_{e e}-w_{i e}+e^{-\frac{t}{\tau_{i}}}\left(w_{e i} /\left(w_{e i}-\theta_{i}\right)\right)^{\frac{\tau_{e}}{\tau_{i}}}+e^{-\frac{t}{\tau_{e}}}\left(w_{e e}+w_{f}-\theta_{e}\right)}{\left(w_{f}-\theta_{e}\right) e^{-\frac{t}{\tau_{e}}}} .
$$

The fixed point $t^{*}$ of the map is related to the traveling pulse solution, i.e. $\xi_{e}^{1}=t^{*}$, and its stability provides the stability of the traveling pulse solution: the traveling pulse solution is stable if $\left|f^{\prime}\left(t^{*}\right)\right|<1$ or equivalently $\left|\left(f^{-1}\right)^{\prime}\left(t^{*}\right)\right|>1$.

To clearly illustrate the role of inhibition and to derive simple tractable conditions for the existence of pulse waves we will focus on different relaxation times. We distinguish between three different cases monitored by the time scale of the inhibitory population. In order to examine the effects of varying $\tau_{i}$ on pulse propagation, we consider the two limiting situations $\tau_{i} \rightarrow \infty, \tau_{i} \rightarrow 0$ that lead to fast-slow systems and turn to the case where $\tau_{i}=\tau_{e}$.

Slow inhibition. We deal with a network with a slow inhibitory process. Study of traveling waves is formally equivalent to the analysis of the singular perturbed system (8) obtained as $\tau_{i} \rightarrow \infty$. Let $\epsilon=1 / \tau_{i}$ be a small positive parameter. In order to determine the expansion of (23) at $\epsilon=0$, we need to consider the following two cases: $\xi_{e}^{1}$ stays constant or becomes a large parameter as $\epsilon \rightarrow 0$. In the first case, that is $\epsilon \xi_{e}^{1}=O(\epsilon)$, the inhibition does not affect the excitatory wave and, from the analysis of the excitatory network, we obtain an unstable traveling pulse. Let us consider the second case where $\epsilon \xi_{e}^{1}=O(1)$. From the leading order expansion of (23) we get

$$
w_{i e} e^{-\epsilon \xi_{e}^{1}}=w_{e e}+w_{i e}+w_{f}-2 \theta_{e}
$$

and we obtain

$$
\xi_{e}^{1}=1 / \epsilon \ln \left(w_{i e} /\left(w_{e e}+w_{i e}+w_{f}-2 \theta_{e}\right)\right) .
$$

Recall that $w_{i e}<0$. To ensure the existence of a positive $\xi_{e}^{1}$, the two following conditions have to be fulfilled

$$
\begin{array}{r}
w_{e e}+w_{f}<2 \theta_{e}-w_{i e} \\
w_{e e}+w_{f}>2 \theta_{e} .
\end{array}
$$

Note that the consistence conditions (24) are fulfilled for sufficiently small $\epsilon$ since $\xi_{e}^{1} \rightarrow+\infty$ as $\epsilon \rightarrow 0$. The linear stability of the traveling pulse is obtained from $\left|\left(f^{-1}\right)^{\prime}\left(\xi_{e}^{1}\right)\right|>1$. We calculate

$$
\begin{aligned}
\left(f^{-1}\right)^{\prime}\left(\xi_{e}^{1}\right) & =\frac{\theta_{e}-w_{e e}-w_{i e}+w_{i e}\left(w_{e i} /\left(w_{e i}-\theta_{i}\right)\right)^{\epsilon \tau_{e}}\left(1-\epsilon \tau_{e}\right) \exp \left(-\epsilon \xi_{e}^{1}\right)}{\theta_{e}-w_{e e}-w_{i e}+w_{i e}\left(w_{e i} /\left(w_{e i}-\theta_{i}\right)\right)^{\epsilon \tau_{e}} \exp \left(-\epsilon \xi_{e}^{1}\right)+\left(w_{f}+w_{e e}-\theta_{e}\right) e^{-\xi_{e}^{1} / \tau_{e}}}, \\
& =1-\epsilon w_{i e} \tau_{e} \exp \left(-\epsilon \xi_{e}^{1}\right) /\left(\theta_{e}-w_{e e}-w_{i e}+w_{i e} \exp \left(-\epsilon \xi_{e}^{1}\right)\right)+O\left(\epsilon^{2}\right) \\
& =1-\epsilon \tau_{e}\left(w_{e e}+w_{i e}+w_{f}-2 \theta_{e}\right) /\left(w_{f}-\theta_{e}\right)+O\left(\epsilon^{2}\right)
\end{aligned}
$$

The traveling pulses are stable if $\left(w_{e e}+w_{i e}+w_{f}-2 \theta_{e}\right) /\left(w_{f}-\theta_{e}\right)<0$. Using condition (26) and the propagation condition $w_{f}>\theta_{e}$, it is clear that the stability condition is fulfilled. 
Figure 8 shows in the parameter plane $\left(w_{e e}, w_{f}\right)$ the regime where a traveling pulse propagates stably in the network. In the presence of slow inhibition, a pulse exists if the total input $w_{e e}+w_{f}+w_{i e}$, obtained when $R_{e}$ and $R_{i}$ reach their maximal values, is not too large; actually lower than a critical value controlled by the excitatory threshold. Moreover the pulse propagates stably if the excitatory feedback $w_{e e}$ is greater than the excitatory threshold. Otherwise the pulse degenerates into a growing pulse as those obtained for the excitatory network.

Fast inhibition. In the case $\tau_{i} \rightarrow 0$ the neural field $r_{i}$ varies on a much faster time scale than $r_{e}$ and inhibitory populations are assumed to act instantly. From (23) the value of $\xi_{e}^{1}$ is given by

$$
\xi_{e}^{1}=\tau_{e} \ln \left[\left(w_{e e}+w_{f}-\theta_{e}\right) /\left(w_{f}+w_{e e}+w_{i e}-2 \theta_{e}\right)\right] .
$$

Using $w_{f}>\theta_{e}$, the existence of $\xi_{e}^{1}$ requires

$$
w_{f}+w_{e e}+w_{i e}>2 \theta_{e}
$$

Under this requirement, the condition $\xi_{e}^{1}>\xi_{i}^{0}$ reads

$$
w_{f}<-w_{e e}+\theta_{e}-w_{e i}\left(w_{i e}-\theta_{e}\right) / \theta_{i} .
$$

The local stability of a traveling pulse solution is given studying the iterated map

$$
t_{k+1}=\tau_{e} \ln \frac{\left(w_{f}-\theta_{e}\right)\left(e^{\frac{t_{k}}{\tau_{e}}}-1\right)-w_{e e}}{\theta_{e}-w_{i e}-w_{e e}} .
$$

The fixed point $t^{*}=\xi_{e}^{1}$ is stable if $\left|f^{\prime}\left(t^{*}\right)\right|<1$. We calculate

$$
f^{\prime}\left(t^{*}\right)=\frac{\left(w_{f}-\theta_{e}\right)\left(\theta_{e}-w_{f}-w_{e e}\right)}{\left(w_{f}-\theta_{e}\right)\left(w_{i e}-\theta_{e}\right)-w_{e e}\left(2 \theta_{e}-w_{i e}-w_{e e}-w_{f}\right)}
$$

that gives

$$
f^{\prime}\left(t^{*}\right)=\frac{w_{f}-\theta_{e}}{\theta_{e}-w_{i e}-w_{e e}}
$$

Recall that $w_{f}>\theta_{e}$. If $w_{e e}+w_{i e}<\theta_{e}$, a pulse wave is stable when $2 \theta_{e}-w_{i e}-w_{e e}-w_{f}>0$. Existence requires (27) and thus we find that a pulse wave is unstable in this regime. To ensure stability we need $w_{e e}+w_{i e}>\theta_{e}$ and the stability condition gives $w_{f}<w_{e e}+w_{i e}$.

Inhibition occurs at $\xi_{i}^{0}$ and induces a jump in the input received by the excitatory population. If the jump triggers the total synaptic input below $\theta_{e}$ then the activity of $R_{e}$ decreases and the inhibition is suddenly stopped. This case leads to a so called sliding solution where the excitatory population slides along $R_{e}=\theta_{i} / w_{e i}$ and the inhibitory wave function has successive jumps. Therefore no traveling pulse exists. Then the following technical condition is necessary to avoid sliding solution

$$
w_{e e} R_{e}\left(\xi_{i}^{0}\right)+w_{i e}+w_{f} R_{e}\left(\xi_{i}^{0}+1 / c_{f}\right)>\theta_{e} .
$$

We have the two following possibilities depending on the relative position of $\xi_{e}^{1}$ and $\xi_{i}^{0}+1 / c_{f}$.

1. For $\xi_{e}^{1}<1 / c_{f}+\xi_{i}^{0}$, we have $R_{e}\left(1 / c_{f}+\xi_{i}^{0}\right)=\left(\exp \left(\xi_{e}^{1} / \tau_{e}\right)-1\right) \exp \left(-\left(1 / c_{f}+\xi_{i}^{0}\right) / \tau_{e}\right)$ and $(29)$ can be expressed as $\left(w_{e e}+w_{i e}-\theta_{e}\right)\left(w_{i e} w_{e i}+w_{e e} \theta_{i}-\theta_{e} w_{e i}-\theta_{e} \theta_{i}+\theta_{i} w_{f}\right) /\left(w_{e i}\left(w_{f}+w_{e e}+w_{i e}-2 \theta_{e}\right)\right)>0$. Using the stability analysis and the result for existence, the jump condition is rewritten $w_{f}>-w_{e e}+\theta_{e}+w_{e i}\left(\theta_{e}-w_{i e}\right) / \theta_{i}$. Since (28) holds there is no solution. 
2. For $\xi_{e}^{1}>1 / c_{f}+\xi_{i}^{0}$, the condition (29) can be written like $w_{e i} w_{f}\left(w_{f}+w_{i e}+w_{e e}-2 \theta_{e}\right)-\left(w_{e i}-\theta_{i}\right)\left(w_{f}-\right.$ $\left.\theta_{e}\right)\left(w_{f}+w_{e e}-\theta_{e}\right)<0$. This condition cannot be fulfilled since $w_{f}<w_{e e}+w_{i e}$.

To conclude, in a network with instantaneous inhibition, traveling pulses do not exist physically, i.e. when they exist they cannot propagate stably.

Identical relaxation times. When the time scale of the inhibitory and excitatory populations significantly differs, it is possible to reduce the model to a rate-model for only one population. This reduction is no longer valid when the two relaxation times are similar. When the excitatory and inhibitory populations have the same relaxation times, $\tau_{i}=\tau_{e}$, the parameter set of the excitatory and inhibitory populations determines the existence and stability of propagating pulses. Solving (23), we get

$$
\xi_{e}^{1}=\tau_{e} \ln \left\{\left(w_{e e}+w_{f}+w_{i e} w_{e i} /\left(w_{e i}-\theta_{i}\right)-\theta_{e}\right) /\left(w_{e e}+w_{i e}+w_{f}-2 \theta_{e}\right)\right\} .
$$

The existence of a traveling pulse is determined by the existence of $c, \xi_{e}^{1}, \xi_{i}^{0}$ and $\xi_{i}^{1}$ that satisfy the apriori assumptions (24). The stability is given studying the iterated map (25) that is written, for $\tau_{e}=\tau_{i}$, as

$$
f(t)=\tau_{e} \ln \frac{\left(w_{f}-\theta_{e}\right)\left(e^{\frac{t}{\tau_{e}}}-1\right)-w_{e e}-w_{i e} w_{e i} /\left(w_{e i}-\theta_{i}\right)}{\theta_{e}-w_{e e}-w_{i e}} .
$$

For convenience we introduce the following notations

$$
\begin{aligned}
\alpha & =w_{f}-\theta_{e}, \\
\beta & =-w_{e e}-w_{i e} w_{e i} /\left(w_{e i}-\theta_{i}\right), \\
\gamma & =\theta_{e}-w_{e e}-w_{i e} .
\end{aligned}
$$

The iterated map is rewritten as $t_{k+1}=\tau_{e} \ln \left[\left(\alpha\left(\exp \left(t_{k} / \tau_{e}\right)-1\right)+\beta\right) / \gamma\right]$ and the fixed point $t^{*}=\xi_{e}^{1}$ is given by $t^{*}=\tau \ln [(\beta-\alpha) /(\gamma-\alpha)]$. The derivative of the map at $t^{*}$ is

$$
f^{\prime}\left(t^{*}\right)=\frac{\alpha}{\gamma}
$$

Note that $\alpha>0$ is the propagation condition. Depending on the sign of $\gamma$ we consider the following two cases:

1. Case $\gamma<0$. The existence of $t^{*}$ reads $\beta<\gamma$ and stability requires $\alpha<-\gamma$. Expressed using the initial parameters we obtain the following conditions: $w_{e e}+w_{i e}>\theta_{e}, \theta_{e} / \theta_{i}>-w_{i e} /\left(w_{e i}-\theta_{i}\right)$ and $w_{f}<w_{e e}+w_{i e}$. We now have to check if the previous conditions are consistent with the apriori assumption $\xi_{e}^{1}>1 / c_{f}$. We get $(\beta-\alpha) /(\gamma-\alpha)>w_{f} /\left(w_{f}-\theta_{e}\right)$ and we derive the following condition $w_{f}<\theta_{e}\left(w_{e i}-\theta_{i}\right) /\left(\theta_{i} w_{i e}\right)\left(w_{e e}-\right.$ $\left.\theta_{e}+w_{i e} w_{e i} /\left(w_{e i}-\theta_{i}\right)\right)$. Combined with previous inequalities, one can show that the conditions on $w_{f}$ do not give admissible solution.

2. Case $\gamma>0$. Existence of $t^{*}$ leads to $\beta>\gamma$ and stability requires $\alpha<\gamma$. We find the following conditions $w_{e e}+w_{i e}<\theta_{e}, w_{f}<2 \theta_{e}-w_{e e}-w_{i e}$ and $\theta_{e} / \theta_{i}<-w_{i e} /\left(w_{e i}-\theta_{i}\right)$. Moreover $\xi_{e}^{1}>1 / c_{f}$ leads to $w_{f}>\theta_{e}\left(w_{e i}-\theta_{i}\right) /\left(\theta_{i} w_{i e}\right)\left(w_{e e}-\theta_{e}+w_{i e} w_{e i} /\left(w_{e i}-\theta_{i}\right)\right)$. In this case a domain can be defined. We have to check the second a priori condition $\xi_{e}^{1}>\xi_{i}^{0}$. It can be written like $(\beta-\alpha) /(\gamma-\alpha)>w_{e i} /\left(w_{e i}-\theta_{i}\right)$. It finally follows $w_{f}>w_{e i} \theta_{e} / \theta_{i}+\theta_{e}-w_{e e}$.

The resulting domain where pulses exist and are stable is represented in Fig. 9. This is a bounded region in the parameter space $\left(w_{e e}, w_{f}\right)$. All the boundaries are analytically calculated as a function of network parameters $w_{e i}, w_{i e}, \theta_{e}$ and $\theta_{i}$. The role of each parameter is clearly illustrated: (i) the stability condition gives the upper bound of the domain and requires that the maximum of the excitatory input $w_{e e}+w_{f}$ is not too large; lower than a quantity given by the excitatory threshold and the I-E connection (ii) the consistency conditions give 
the lower bound of the domain that is determined by a subtle combination of the E-I connection and the ratio between the two thresholds (iii) an additional condition, that does not involved the two synaptic connections $w_{e e}, w_{f}$, gives a maximal value for the thresholds ratio.

The propagation of a traveling pulse in the balanced network is depicted in Fig. 10. The numerical simulation is done using a finite chain of pools. From the profile of the iterative map, it is sufficient to apply a superthreshol input to the first pool with a time duration greater than $t^{*}$ in order to elicit the propagation of a pulse. Since a single iteration is related to a transition from one pool to the next, the convergence of an initial activation to the traveling pulse is achieved according to the global shape of the iterative map.

Similarly to the excitatory network, the region of existence of traveling pulses is enclosed in the traveling front and traveling back domain. However in balanced networks the stability criteria and the existence conditions of pulse waves are not mutually exclusive. More precisely the existence of a stable traveling pulse precludes the existence of a growing pulse. In fact for large $t_{k}$ values we have $t_{k+1}-t_{k}=\tau_{e} \ln \left(\left(w_{f}-\theta_{e}\right) /\left(\theta_{e}-w_{e e}-w_{i e}\right)\right)$. Pulse stability requires $w_{f}<2 \theta_{e}-w_{e e}-w_{i e}$ and thus we have $t_{k+1}-t_{k}<0$, i.e. the interval of excitation decreases and stabilizes to the traveling pulse one (see Fig. 10).

\subsection{Robustness and limitations}

In this section the generality of the results are tested through numerical simulations. In particular, one might question the robustness of the results with regard to the limiting cases considered for the relaxation times and for the transfer function.

The dependence of the stable traveling pulse on $\tau_{i}$ is shown in fig. 11 for various parameters, together with results of stability. We represent the rising interval of the excitatory wave, $\xi_{e}^{1}$, as a function of $\tau_{i}$. Parameters are such that a traveling pulse exists when $\tau_{i}=\tau_{e}$. In agreement with our analysis, traveling pulse exists for large $\tau_{i}$ and disappears as $\tau_{i} \rightarrow 0$. Numerically, we find that there is a critical relaxation time $\tau_{i}^{*}$ below which traveling pulse fails to propagate. Different scenarios are encountered: (I) Traveling pulse destabilizes into a growing pulse due to the apparition of an additional bump in the tail of the wave (the excitatory traveling pulse solution $R_{e}$ presents two distinct rising intervals). (II) The condition $\xi_{e}^{1}>1 / c_{f}$ is violated at $\tau_{i}=0.90$ and propagation failure occurs. (III) The traveling pulse presents a sliding regime, i.e. $R_{e}=\theta_{i} / w_{e i}$ on a non-empty interval, that leads to an enlarging pulse. Note that both destabilizations (I) and (III) are not predicted by the study of the iterative map (25) that only addresses stability of one pulse wave. The instabilities associated with global bifurcations where pools reached the threshold $\theta_{e}$ from below more than once are not captured by our stability analysis.

We perform numerical simulations of the network with the smooth activation function (2). For large gain, results are in agreement with our study. Figure 12 shows the propagation of a traveling pulse using a gain $\lambda=20$ for the activation function and other parameters as in fig. 10 .

\section{Discussion}

We analyze traveling waves in a feedforward neural network where synaptic connections are made only from one pool to the next and excitation is balanced by inhibition. From a mathematical point of view, our model is a set of differential equations on a lattice and the problem of finding traveling wave solutions leads to an advanced differential equation for which the theory is recent [16], [4]. Because our model is piecewise linear we are able to derive analytically the properties of propagating waves. We have constructed the traveling wave solutions and derived precise conditions for their existence and stability. We use the techniques from bifurcation theory to characterize front and back waves. The two saddle node bifurcations of an isolated pool allow for a qualitative understanding of the mechanisms that lead to propagating waves in the connected network. The propagation of a front wave and a back wave occurs when the corresponding saddle node bifurcation observed in the one-pool model is reached. The time to reach the first bifurcation is related to the velocity of the back $c_{b}$ whereas the second is related to the velocity the front, $c_{f}$. Traveling fronts and traveling backs are robust in the sense that 
a limited number of conditions is sufficient to ensure their existence: they are poorly affect by the exact nature of the model and only require a bistability condition, i.e. the existence of low or high activity steady state for the network.

The purely excitatory network does not support the propagation of stable pulses. We have shown that traveling pulses are unstable because of the existence of stable front waves and stable back waves with different velocities. This mechanism presents interesting links with the absolute instabilities reported in [21]. A "reverse" property is obtained in [20] and [22] where a stable pulse is obtained when merging unstable front and back. In our network, adding inhibition allows the propagation of pulses with a constant shape along the network. When excitation is balanced appropriately by inhibition the growing pulse observed in the excitatory network is suppressed and an initial pulse converges towards the traveling pulse solution. The regime where a traveling pulse propagates depends strongly on the kinetic of the inhibitory population. In the limit of instantaneous inhibition, pulse waves disappear. When the two populations have identical time scales, the regime of existence and stability of pulses depends on the entire parameter set of the network. Increasing the passive time constant of inhibitory cells gives a regime where traveling pulses propagate stably that depends only on the parameters of the excitatory pools and the inhibitory feedback (the I-E connection).

\section{References}

[1] M. Abeles, Corticonics. Cambridge: Cambridge University Press, 1991.

[2] S.I. Amari, Dynamics of pattern formation in lateral-inhibition type neural fields, Biol. Cybern. 27 (1977) $77-87$.

[3] P.C. Bressloff, Synaptically generated wave propagation in excitable neural media, Phys. Rev. Lett. 82 (1999) 2979-2982.

[4] S.N. Chow, J. Mallet-Paret, Traveling waves in lattice dynamical sytems, J. Diff. Eq. 149 (1998) 248-291.

[5] S. Coombes, M.R. Owens, Evans functions for integral neural field equations with Heaviside firing rate function, SIAM J. Appl. Dyn. Syst. 3 (2004) 574-600.

[6] M. Diesmann, M.O. Gewaltig, A. Aertsen, Stable propagation of synchronous spiking in cortical neural networks, Nature 402 (1999) 529-533.

[7] M. Enculescu, A note on traveling fronts and pulses in a firing rate model of a neuronal network, Physica D 196 (2004) 362-386.

[8] G.B. Ermentrout, The analysis of synaptically generated traveling waves, J. Comput. Neurosci. 5 (1998) 191-208.

[9] G.B. Ermentrout, J.B. McLeod, Existence and uniqueness of travelling waves for a neural network, Proc. Roy. Soc. Edinburgh A 123 (1993) 461-478.

[10] S.E. Folias, P.C. Bressloff, Breathing pulses in an excitatory neural network, SIAM J. Appl. Dyn. Syst. 3 (2004) 378-407.

[11] F. Giannakopoulos, K. Pliete, Planar systems of piecewise linear differential equations with a line of discontinuity, Nonlinearity 14 (2001) 1611-1632.

[12] D. Golomb, Y. Amitai, Propagating neuronal discharges in neocortical slices: computational and experimental study, J. Neurophysiol. 78 (1997) 1199-1211.

[13] U. Kim, T. Bal, D.A. McCormick, Spindle waves are propagating synchronized oscillations in the ferret LGNd in vitro, J. Neurophysiol. 74 (1995) 1301-1323. 
[14] W.M. Kistler, W. Gerstner, Stable propagation of activity pulses in populations of spiking neurons, Neural Comput. 14 (2002) 987-997.

[15] V. Litvak, H. Sompolinsky, I. Segev, M. Abeles, On the transmission of rate code in long feedforward networks with excitatory-inhibitory balance, J. Neurosci. 23 (2003) 3006-3015.

[16] J. Mallet-Paret, The global structure of traveling waves in spatially discrete dynamical systems, J. Dyn. Diff. Eq. 11 (1997) 49-127.

[17] R. Miles, R.D. Traub, R.K.S. Wong, Spread of synchronous firing in longitudinal slices from the CA3 region of the hippocampus, J. Neurophysiol. 60 (1988) 1481-1496.

[18] R. Osan, G.B. Ermentrout, The evolution of synaptically generated waves in one and two-dimensional domains, Physica D 163 (2002) 217-235.

[19] D.J. Pinto, G.B. Ermentrout, Spatially structured activity in synaptically coupled neuronal networks:I. Traveling fronts and pulses, SIAM J. Appl. Math. 62 (2001) 206-225.

[20] M.M. Romeo, C.K.R.T. Jones, Stability of neuronal pulses composed of concatenated unstable kinks, Phys. Rev. E 63 (2001) 011904.

[21] B. Sandstede, A. Scheel, Absolute and convective instabilities of waves on unbounded and large bounded domains, Physica D 145 (2000) 233-277.

[22] B. Sandstede, A. Scheel, Gluing unstable fronts and backs together can produce stable pulses, Nonlinearity 13 (2000) 1465-1482.

[23] M.C.W. van Rossum, G.G. Turrigiano, S.B. Nelson, Fast propagation of firing rates through layered networks of noisy neurons, J. Neurosci. 22 (2002) 1956-1966.

[24] H.R. Wilson, J.D. Cowan, A mathematical theory of the functional dynamics of cortical and thalamic nervous tissue, Kybernetik 13 (1973) 55-80.

[25] L. Zhang, On the stability of traveling wave solutions in synaptically coupled neuronal networks, Diff. Int. Eq. 16 (2003) 513-536. 


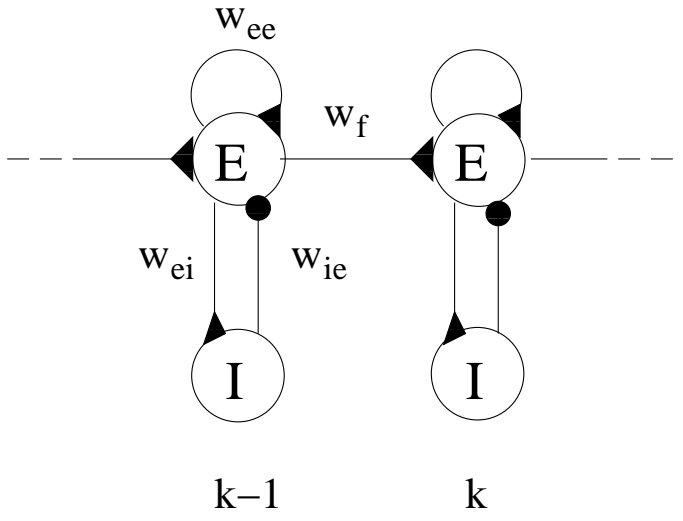

Figure 1: The schematic representation of the one-dimensional feedforward network with local inhibition (I) and excitatory (E) coupling. The pool at a location $k$ receives an excitatory effect from the activity of the pool at the location $k-1$. 


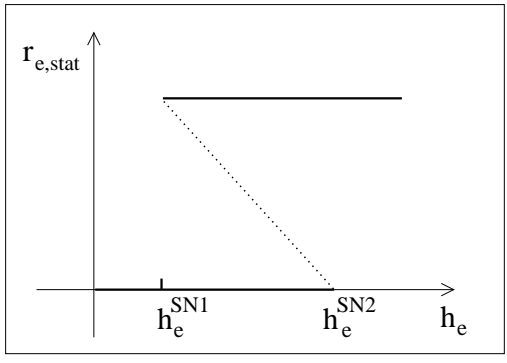

A

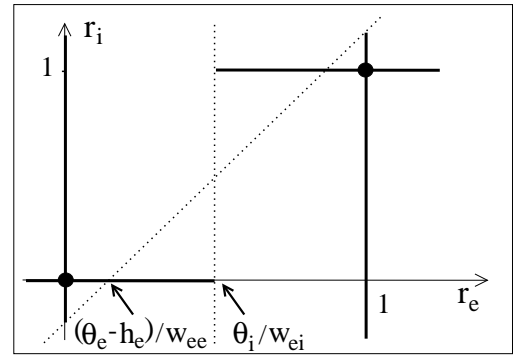

$\mathrm{B}$

Figure 2: Analysis of the single pool model. The bifurcation diagram in panel A shows the steady-state firing rate of the excitatory population $r_{e, \text { stat }}$ versus the external input $h_{e}$. Panel B shows the two-dimensional phase plane $\left(r_{e}, r_{i}\right)$ of a single pool in the case $w_{e i}>\theta_{i}$ referred as the balanced network. Domains where the system is linear are delimited by the threshold conditions (dotted lines). Intersections of the threshold lines with the $r_{e}-$ axis are indicated. Nullclines of the system, i.e. $d r_{e} / d t=0$ and $d r_{i} / d t=0$, are represented (solid lines) for the bifurcation parameter $h_{e}$ that satisfies $h_{e}^{\mathrm{SN} 1}<h_{e}<h_{e}^{\mathrm{SN} 2}$. The system has two stable fixed points: a low-activity steady state $(0,0)$ and a high-activity steady state $(1,1)$ represented by filled black circles. 


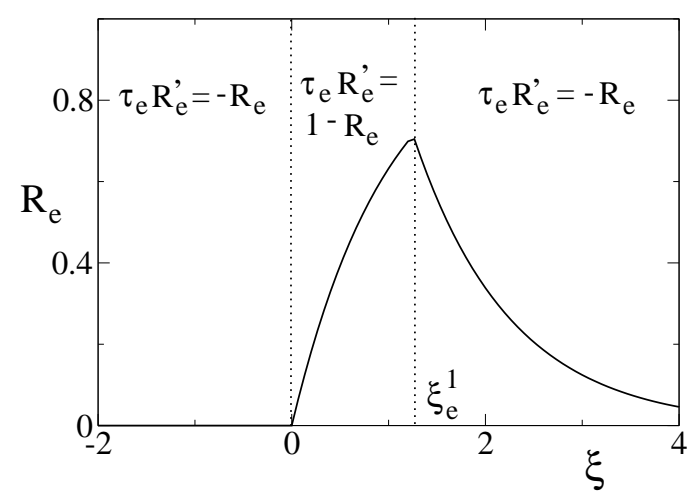

Figure 3: The traveling pulse of firing rate activity in the excitatory neural network. The dynamics of the wave is decomposed into a rising phase, $\xi \in\left(0, \xi_{e}^{1}\right)$, and a decaying phase, $\xi \in\left(\xi_{e}^{1},+\infty\right)$. Parameters are $\tau_{e}=1$, $w_{e e}=0.2, \theta_{e}=0.5$ and $w_{f}=1$. We calculate the wave speed $c_{f}=1.44$ and the so-called inactivation point $\xi_{e}^{1}=1.25$. The traveling pulse (constant profile) is unstable leading to a pulse propagation with an enlarging profile. 


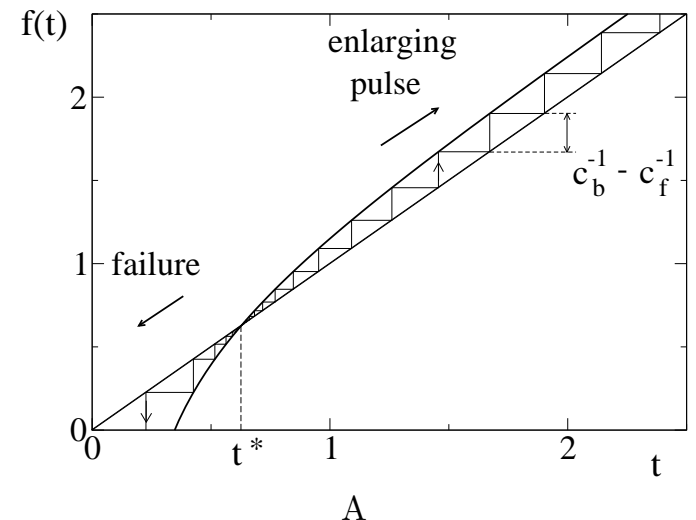

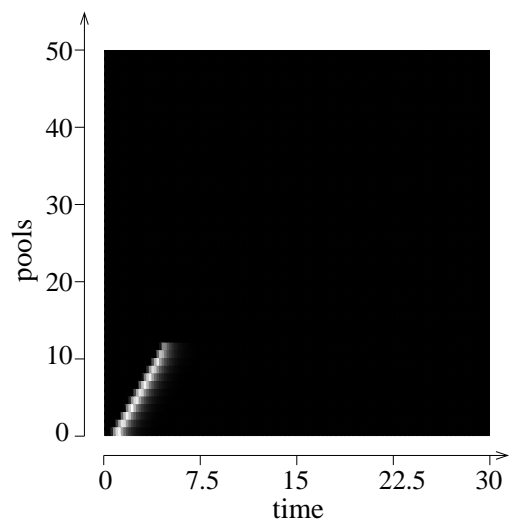

$\mathrm{B}$

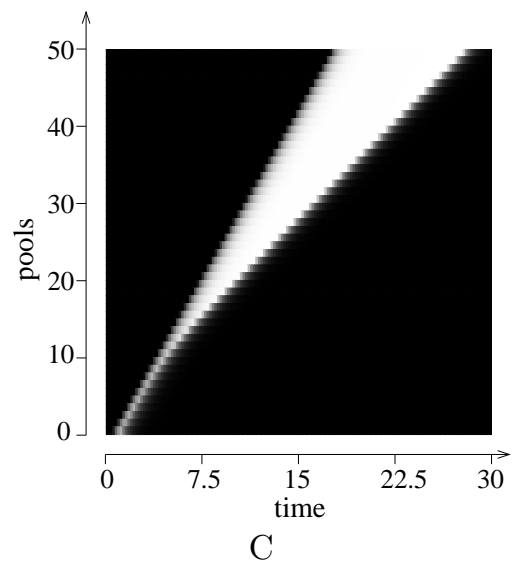

C

Figure 4: (A) The iterated map $t_{k+1}=f\left(t_{k}\right)$ that gives the evolution of pulses. The fixed point $t^{*}$ is related to the width of the traveling pulse solution, i.e. a pulse with constant shape. The fixed point is unstable and, depending on the initial excitation, propagation failure occurs after a transient propagation (B) or an enlarging pulse propagates due to the destabilization of the traveling pulse (C). Panels (B) and (C) are obtained from the numerical simulation of a network of 50 pools (vertical axes) during 30 units of (normalized) time (horizontal axes) with different initial conditions. The initial pulse has a rising interval lower than $t^{*}(\mathrm{~B})$ and greater in (C). Parameters are $\tau_{e}=0.5, w_{e e}=0.2, \theta_{e}=0.5$ and $w_{f}=1$. We calculate $t^{*}=0.62$ and $f^{\prime}\left(t^{*}\right)=1.67$. 

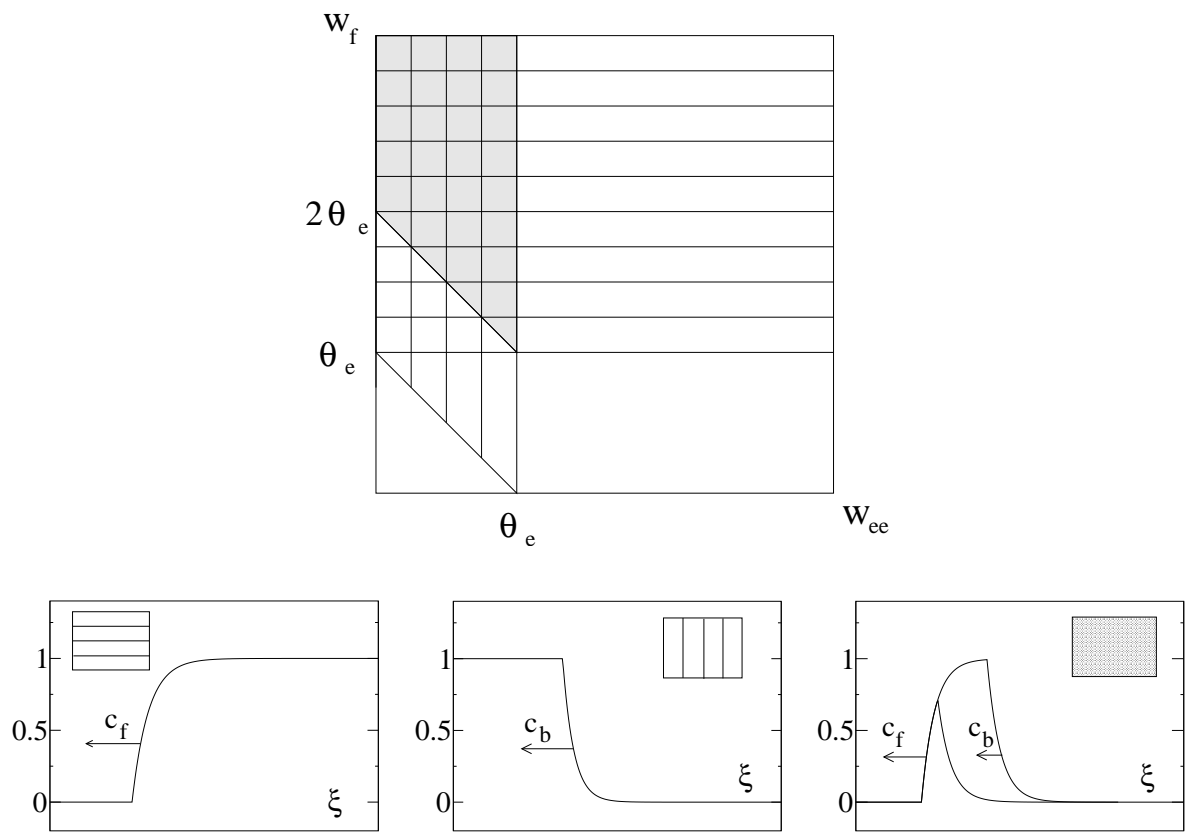

Figure 5: (top) Locus of existence of traveling waves in the $\left(w_{e e}, w_{f}\right)$ plane for the excitatory network. The corresponding traveling waves (indicated by a small inserting box) are depicted below. Horizontal lines indicate the existence of front waves, vertical lines denote back waves and solid shading indicates pulses. The speed $c_{f}$ is the velocity of a front and $c_{b}$ is the velocity of a back wave. The traveling pulse is unstable leading to an enlarging pulse with a velocity of the front, $c_{f}$, and a velocity of the wake, $c_{b}$, that are different, $c_{f}>c_{b}$. 

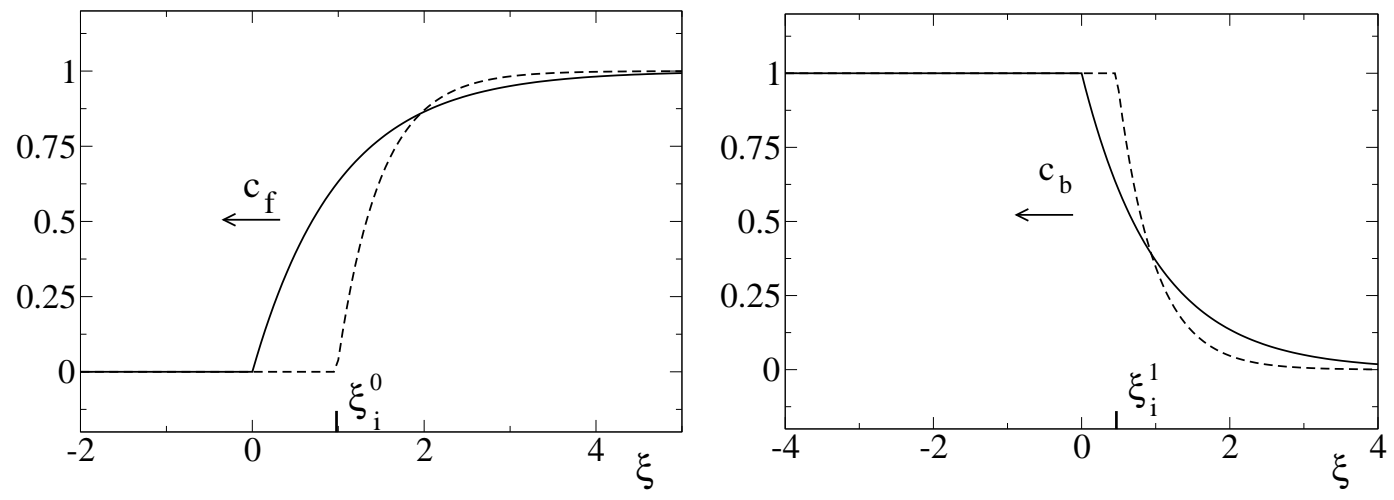

Figure 6: The wave profile of the traveling front (left panel) and the traveling back (right panel) in the balanced network. The profile of the excitatory (solid line) and inhibitory (dashed line) traveling waves are shown as a function of the traveling wave coordinate $\xi$. For the traveling front, the inhibitory population is activated at $\xi=\xi_{i}^{0}$. The activity of the inhibitory population decreases at $\xi=\xi_{i}^{1}$ for the traveling back solution. Parameters are $w_{e e}=1, w_{i e}=-0.7, w_{e i}=0.8 w_{f}=0.6, \theta_{e}=0.5, \theta_{i}=0.5, \tau_{e}=1$ and $\tau_{i}=0.5$. We calculate $\xi_{i}^{0}=0.98$ and $\xi_{i}^{1}=0.47$. 


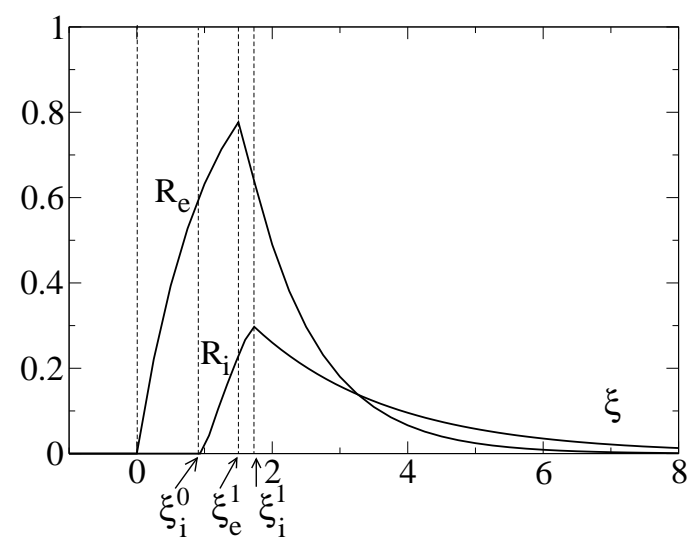

Figure 7: The traveling pulse solution in the balanced network. The excitatory $R_{e}$ and inhibitory $R_{i}$ wave functions are represented in the traveling wave coordinate $\xi$. Parameters are $\tau_{e}=1, \tau_{i}=2, w_{e e}=0.4$, $w_{i e}=-1.5, w_{e i}=0.8, \theta_{e}=\theta_{i}=0.5$ and $w_{f}=1.2$. We calculate $c=1.44$ and $\xi_{e}^{1}=1.53, \xi_{i}^{0}=0.98$ and $\xi_{i}^{1}=1.7$. 


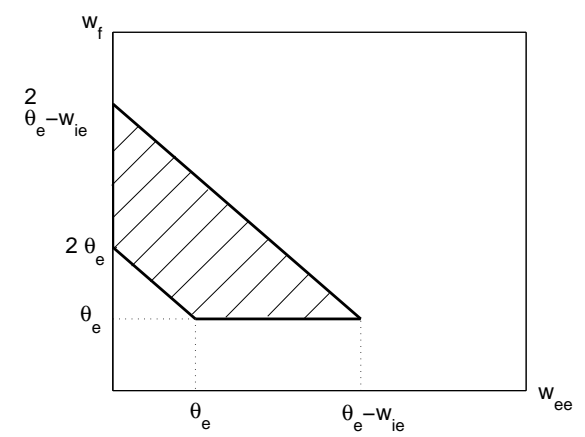

Figure 8: Regime of existence and stability of traveling pulse solutions in the $\left(w_{e e}, w_{f}\right)$ plane for a balanced network with slow inhibition, i.e. $\tau_{i} \rightarrow \infty$. 


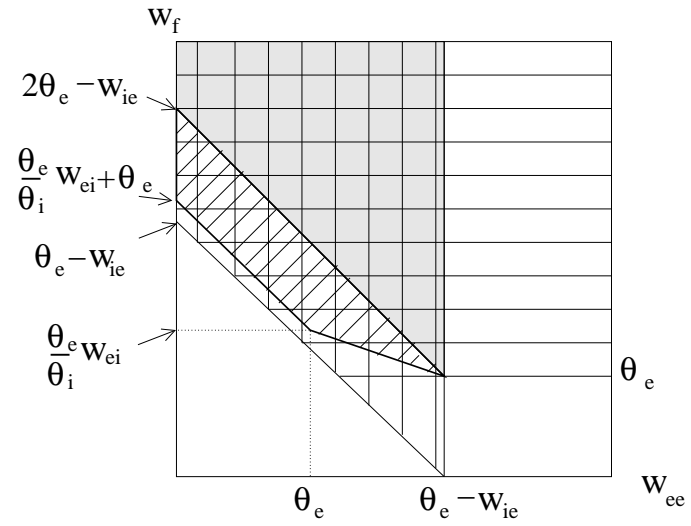

Figure 9: Regime of existence and stability of traveling wave solutions in the $\left(w_{e e}, w_{f}\right)$ plane for the balanced network where the two populations have identical relaxation times. An additional condition that is $\theta_{e} / \theta_{i}<$ $-w_{i e} /\left(w_{e i}-\theta_{i}\right)$ does not appear explicitly in the diagram but has to be fulfilled to ensure the stability of the pulse wave. As in fig. 5 the regime of existence of a traveling wave front is indicated by horizontal lines, traveling backs with vertical lines and growing pulses with solid shading. Dashed lines indicate the new regime of existence and stability of the traveling pulse solutions. Propagation failure occurs in the other regions. 


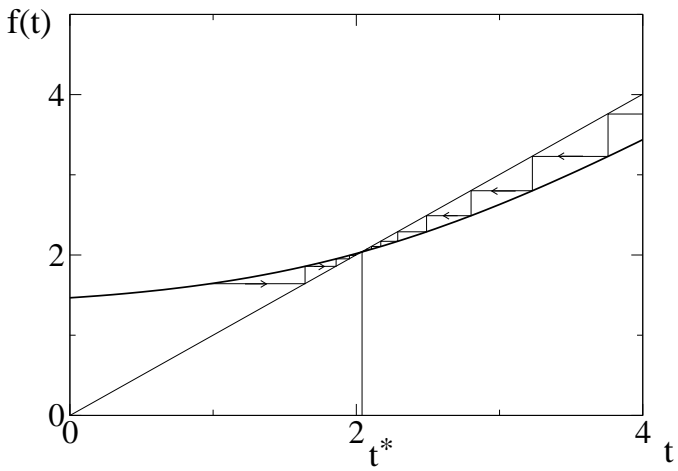

A

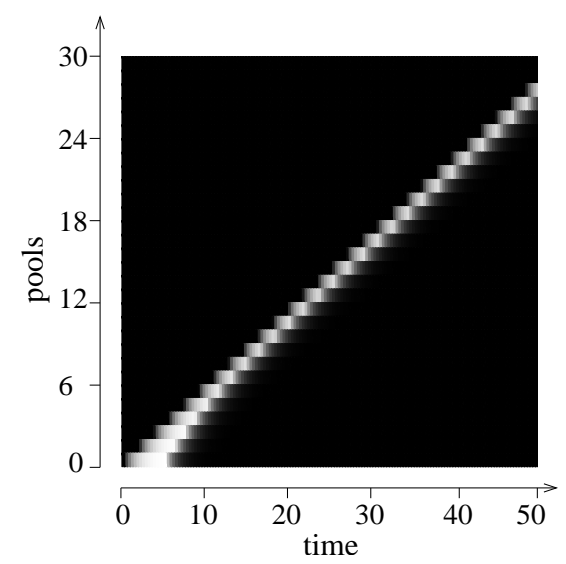

$\mathrm{B}$

Figure 10: Traveling pulse in a balanced network. (A) The iterated map that predicts the dynamics of traveling pulses. The fixed point $t^{*}$ represents the rising interval of the traveling pulse solution. Panel (B) shows the propagation of the corresponding pulse wave obtained from the simulation of a network of 30 (vertical axes) pools during 50 units of normalized time. The initial excitation is a square input of duration 5 (greater than $t^{*}$ ). Parameter values are $\tau_{e}=\tau_{i}=1, w_{e e}=1, w_{e i}=0.8, w_{i e}=-0.7, \theta_{e}=\theta_{i}=0.5$ and $w_{f}=0.6$. We calculate $t^{*}=2.04$ and $f^{\prime}\left(t^{*}\right)=0.5$. 


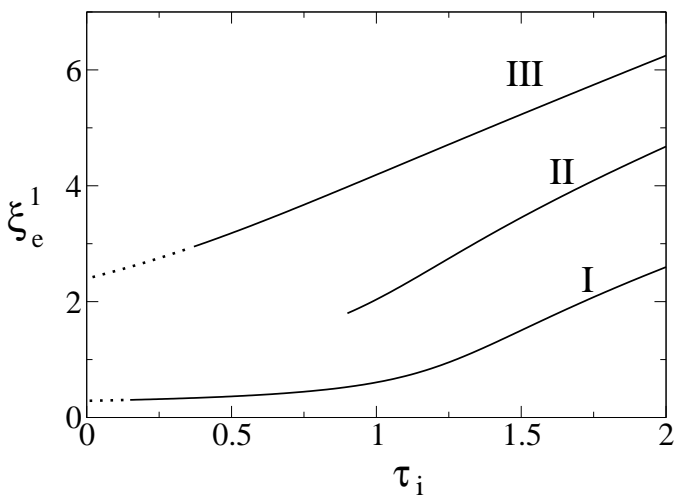

Figure 11: Rising interval of the excitatory traveling pulse, $\xi_{e}^{1}$, as a function of the relaxation time of the inhibitory population, $\tau_{i}$, for several values of parameters. Solid lines represent stable traveling pulse solutions and dotted lines denote unstable traveling pulses. Parameters $\left(\tau_{e}, w_{e e}, w_{e i}, w_{i e}, w_{f}, \theta_{e}, \theta_{i}\right)$ are (I) $(1,2,2,-4,2,0.5,0.5)$, (II) $(1,1,0.8,-0.7,0.6,0.5,0.5)$ and (III) $(1,0.6,0.55,-0.7,1,0.5,0.5)$. There is a critical relaxation time $\tau_{i}^{*}$ below which traveling pulse fails to propagate or is unstable. We calculate (I) $\tau_{i}^{*}=0.14$, (II) $\tau_{i}^{*}=0.90$ and (III) $\tau_{i}^{*}=0.36$. 


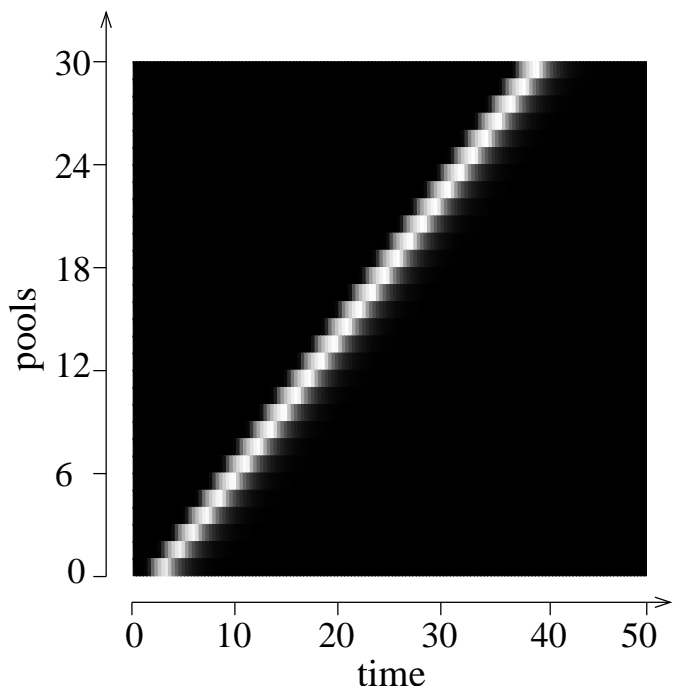

Figure 12: Propagation of a pulse wave in a network of 30 (vertical axes) pools during 50 units of normalized time. A sigmoidal activation function is used with a gain $\lambda=20$. Other parameters are as in fig. 10b. 